\title{
Sulcal Fluid-Attenuated Inversion Recovery Hyperintensity After Cerebrospinal Fluid Removal in Intracranial Hypertension?
}

\author{
Halil Onder
}

\section{To the Editor}

I read with great interest the article by Yazdani et al, published in the latest issue of European Journal of Radiology Open, in which they illustrate the finding of sulcal fluid-attenuated inversion recovery (FLAIR) hyperintensity as a neuroimaging sign of intracranial hypotension after cerebrospinal fluid (CSF) removal [1]. In conclusion, the authors remark the importance of awareness of this potential cause for sulcal FLAIR hyperintensity in the appropriate patient population for prevention of further unnecessary workup for other etiologies. I appreciate the authors for illustrating such an original and potentially useful finding as well as concluding a critical message to be kept in mind in the clinical practice. However, there may be some additional points to be discussed.

Sulcal hyperintensity on FLAIR imaging has generally been considered of abnormal CSF, such as meningitis and subarachnoid hemorrhage [2]. The authors remark a previous study, showing that cortical sulcal CSF accounts for the majority of reduced CSF volume following lumbar puncture [3]. Besides, they propose a mechanism that sulcal FLAIR hyperintensity is possibly due to a decrease in the ratio of CSF according to blood pool which becomes the dominant contributor to sulcal FLAIR signal (as also reported by Taoka et al [2]) in their reported patients [1]. At this point, I would like to remark that the total amount of CSF produced in 24 $\mathrm{h}$ is about $600 \mathrm{~mL}$ and it is known that the total volume is replaced about four times each day [4]. Hence, based on this rapid circulation process of CSF, it can be understood that in a considerable amount of the etiologies of intracranial hypotension which are subacute or chronic, hemostasis and the balance between the intracranial components (brain, blood, and CSF) is rather established. Therefore, this mentioned finding of sulcal FLAIR hyperintensity (which has been hypothesized to represent an acute alteration of sulcal content via a mechanism of intracranial pressure (ICP) compensation due to rapid CSF pressure decrement) might not occur due to

Manuscript submitted February 9, 2019, accepted March 7, 2019

Neurology Clinic, Yozgat City Hospital, Yozgat, Turkey.

Email: halilnder@yahoo.com

doi: https://doi.org/10.14740/jnr523 the rather more gradual compensatory mechanisms proceeding in most of the patients with intracranial hypotension. In conclusion, I would like to propose that this finding might be attributed to a mechanism of rapid decrease of intracranial CSF (rather than to associate primarily with intracranial hypotension) with a resulting reduction in the volume-pressure relationship and volume compensation due to an increase in intracranial blood volume, which was also hypothesized previously [3]. Future studies investigating the presence of this particular neuroimaging sign after lumbar puncture (LP) in patients with intracranial hypertension and also normotensive ICP, may give substantial perspectives regarding the underlying mechanisms. In addition, the comparative investigation of the occurrence of this appearance according to the temporal properties (acute or chronic) of the disease (intracranial hypotension) may provide contributions in this regard. The results of these studies may provide potentially a crucial clinical remark such as avoiding rapid decrement of ICP in patients with higher LP opening pressures, to prevent disturbing the brain adaptation to high $\mathrm{CP}$, as stated similarly to hyponatremia in osmotic demyelination syndrome [5]. Finally, I think that sulcal FLAIR hyperintensity, representing the volume compensation by an increase in the intracranial blood volume [3] induced by CSF volume loss, may potentially be a neuroimaging correlate of post LP headache, which was similarly hypothesized by Grant et al [3]. Future reports addressing these mentioned points may develop our understanding of the disturbed as well as normal dynamics of CSF physiology.

\section{Acknowledgments}

None.

\section{Conflict of interest}

None.

\section{Funding}

None. 


\section{Informed Consent}

The informed consent form is not applicable.

\section{Author Contributions}

Halil Onder contributed to all the stages (to conception and design, and/or acquisition of data, and/or analysis and interpretation of data, drafting the article or revising it critically for important intellectual content, etc.) during preparing this manuscript.

\section{References}

1. Yazdani M, Stalcup ST, Chatterjee AR, Matheus MG.
Sulcal FLAIR hyperintensity after CSF removal in two patients with intracranial hypertension. Eur J Radiol Open. 2019;6:33-35.

2. Taoka T, Yuh WT, White ML, Quets JP, Maley JE, Ueda $\mathrm{T}$. Sulcal hyperintensity on fluid-attenuated inversion recovery $\mathrm{mr}$ images in patients without apparent cerebrospinal fluid abnormality. AJR Am J Roentgenol. 2001;176(2):519-524.

3. Grant R, Condon B, Hart I, Teasdale GM. Changes in intracranial CSF volume after lumbar puncture and their relationship to post-LP headache. J Neurol Neurosurg Psychiatry. 1991;54(5):440-442.

4. Wright EM. Transport processes in the formation of the cerebrospinal fluid. Rev Physiol Biochem Pharmacol. 1978;83:3-34.

5. Sterns RH, Hix JK, Silver S. Treatment of hyponatremia. Curr Opin Nephrol Hypertens. 2010;19(5):493-498. 